\title{
Proposta de Implementação de uma Ferramenta Gráfica para Geração de Relatórios PDF com PHP e FPDF
}

\author{
Igor H. Berlitz \\ igor.berlitz@terra.com.br
}

\begin{abstract}
Resumo. Este artigo apresenta uma proposta de ferramenta gráfica para geração de arquivos no formato PDF, implementado para ser acessado via Web e construído sobre a classe FPDF escrita em PHP. O sistema proposto atende as necessidades básicas para a criação e exportação de relatórios Web no formato PDF, possibilitando que o usuário possa criar seu próprio relatório através do navegador de forma simples e ágil.
\end{abstract}

\section{Introdução}

Nunca a informação foi tão importante para a economia. Em um mercado globalizado e competitivo, onde cada vez mais se propaga a idéia de que uma empresa é essencialmente uma processadora de informações, o papel da informação como uma ferramenta essencial para o gerenciamento e funcionamento das organizações torna-se cada vez mais claro, permitindo análise estratégica e auxílio nas decisões (Feraud APUD Davenport, Dickson e Marchand 2004).

Nesse contexto, a Tecnologia da Informação (TI) que durante algum tempo foi considerada apenas como um item de suporte aos processos administrativos, hoje passa a ser encarada como um importante recurso, que agrega valor ao produto ou serviço final, conseqüentemente, oferecendo um diferencial competitivo.

O surgimento de inovações em termos de TI cresceu de forma substancial nos últimos anos, com destaque para a Internet, que se constitui atualmente no conjunto de TI com maior impacto na sociedade. A popularização da Internet e o surgimento dos sistemas Web trouxeram inúmeras vantagens, como a possibilidade de acesso em qualquer lugar do planeta, a capacidade de utilização em todas as plataformas que suportem um navegador HTTP e facilidade de atualização. Nos dias atuais, a maioria dos pacotes de software das mais diferentes áreas, desde pequenas aplicações à grandes sistemas de gestão, utilizam esse tipo de interface.

Em função do crescimento das aplicações Web, o número de informações e relatórios nas organizações tomou proporções gigantescas, mas com um problema ser resolvido: a disponibilização em formatos nem sempre fáceis de utilizar. Dessa forma, ao necessitar de uma resposta os tomadores de decisão, em muitos casos, dependem da ajuda do pessoal de TI (SAS 2006).

Para Thomson e Welling (2003), uma forma de resolver esse problema é a geração desses relatórios no formato de arquivo PDF, uma vez que esse tipo de arquivo oferece saída consistente e de alta qualidade, extremamente útil na distribuição de documentos imprimíveis e atraentes, características de extrema importância em aplicações Web. 
Este presente estudo descreve uma proposta de ferramenta Web a ser desenvolvida na linguagem PHP e utilizando como base a classe FPDF, no intuito de auxiliar o usuário final na montagem e personalização dos relatórios a serem gerados no formato PDF.

Este artigo está dividido em seções que abordarão alguns conceitos relevantes ao domínio deste trabalho. Na seção 2 serão apresentadas bibliotecas PHP para geração de relatórios PDF e um estudo mais aprofundado da biblioteca FPDF, mostrando suas vantagens e desvantagens. Na seção 3 serão apresentados conceitos e definições de AJAX e sua forma de funcionamento. Na seção 4 a proposta da ferramenta é descrita, detalhando sua arquitetura, estrutura e funcionamento. E por fim, na seção 5 são apresentadas as considerações finais.

\section{Bibliotecas PHP para geração de relatórios PDF}

Segundo Thomson e Welling (2003), o PHP disponibiliza suporte nativo para a criação de documentos PDF através de duas bibliotecas de funções diferentes, mas com intenções semelhantes. As bibliotecas são a PDFlib (PDFlib 2006) e a ClibPDF (PHP 2006), que contam com bibliotecas externas e não são compiladas para o PHP por padrão. Essas duas bibliotecas fornecem uma API de funções que geram os documentos no formato PDF, podendo ser utilizadas sem custo para uso pessoal, mas exigem o pagamento de taxa de licença se forem utilizadas em aplicações comerciais.

Fuecks (2003) explica que outra forma de geração de documentos PDF com PHP é a utilização das classes R\&OS PDF Class (R\&OS 2006) e FPDF (FPDF 2006). Diferentemente da PDFlib e da ClibPDF, essas duas não são bibliotecas propriamente ditas e sim classes escritas em PHP. Essas classes são gratuitas, portanto para utilizá-las basta fazer uma cópia dos seus arquivos no servidor Web e efetuar a chamada no início dos programas.

Foi escolhida a utilização da classe FPDF para o desenvolvimento da ferramenta, por se tratar de uma classe de simples manuseio que possui inúmeras funcionalidades a serem exploradas, seja de maneira direta ou através de suas extensões. Outro fator de extrema importância para sua escolha é o fato de ser uma classe gratuita que possibilita sua utilização tanto para o uso pessoal como comercial, permitindo um estudo mais aprofundado do seu código-fonte e de possíveis modificações conforme a necessidade do desenvolvedor.

\subsection{FPDF}

Como o sistema foi desenvolvido tomando como base a classe FPDF, será descrita a terminologia utilizada pela mesma, assim como suas vantagens e desvantagens.

\subsection{O que é FPDF?}

FPDF é uma classe PHP gratuita, desenvolvida por Olivier Plathey, para geração de arquivos PDF em PHP. Tem como principais características: permitir a escolha do formato da página e tamanho das margens, quebra automática de página, quebra automática de linha e alinhamento de página justificado, suporta imagens nos formatos JPEG e PNG, possibilita colorir o texto e incluir links (FPDF 2006). Para utilizá-la basta baixar o código-fonte do site do desenvolvedor, extrair a FPDF em diretório no 
servidor, e por fim incluir a classe no código PHP indicando o caminho em que se encontra a classe.

\subsection{Vantagens}

Podem-se destacar as seguintes vantagens da utilização da classe:

- Classe gratuita que permite utilização tanto para uso pessoal como comercial;

- Funciona em qualquer servidor PHP;

- Possui métodos que podem ser sobrescritos, permitindo a herança de classes e implementação de métodos próprios;

- Não necessita de extensões.

\subsection{Desvantagens}

As desvantagens que levaram ao estudo mais aprofundado da implementação de uma ferramenta que auxilia geração de arquivos PDF com a ferramenta FPDF são as seguintes:

- Toda a montagem do relatório é feita via programação PHP, não permitindo que o usuário possa criar o relatório conforme suas necessidades;

- Necessita definir manualmente no código PHP a formatação e posicionamento do texto e de todos os elementos que formam o relatório;

- Não suporta texto na diagonal.

\section{AJAX}

Um dos grandes responsáveis na implementação da ferramenta proposta, em sua interface com o usuário, é a tecnologia AJAX, relacionada à comunicação e transferência de dados entre cliente e servidor.

AJAX, acrônimo de Asyncronous Javascript and XML, é o uso sistemático de Javascript e XML com o intuito de transformar o navegador mais interativo com o usuário. O termo AJAX foi cunhado por Jessé James Garret, e ganhou popularidade ao mostrar-se um bom termo para descrever técnicas que permitem aplicações Web interagir com um servidor assincronamente. Segundo Garret (2005), o AJAX não é uma tecnologia, são na realidade várias tecnologias progredindo de forma independente, e que se juntaram a fim de explorar formas de melhorar a interação com os usuários em aplicações Web.

AJAX incorpora no seu modelo as seguintes tecnologias (Wikipedia 2006):

- Padronização da apresentação baseada em XHTML (Niederaurer 2001) e CSS (Teague 2001);

- Exposição e interação dinâmica usando o DOM (Ray 2001);

- Intercâmbio e manipulação de dados usando XML (Daum 2002) e XSLT (Wikipedia 2006a); 
- Recuperação assíncrona de dados usando o objeto XMLHttpRequest (Asleson e Schutta 2006); e

- Javascript (Negrino e Smith, 2004) fazendo a integração dos anteriores.

No modelo tradicional das aplicações Web a maioria das ações do usuário envia uma solicitação HTTP para o servidor Web, que faz o processamento, e então devolve uma página HTML por completo (Garret 2005). O grande problema desse modelo é a necessidade de, em cada solicitação, executar novamente todo o processo de envio, criar uma página e devolver ao navegador, que sempre recebe uma página completa. Estas atividades geram um tráfego de rede excessivo e envio desnecessário de dados que não foram alterados. A conseqüência deste processo para o usuário, muitas vezes, é uma tela muito lenta e páginas em branco.

O modelo AJAX adiciona uma camada intermediária entre o cliente e o servidor. A interface ao invés de enviar uma solicitação HTTP lança uma chamada de Javascript para uma aplicação AJAX que se encontra do lado do cliente. Esta, por sua vez, faz um pedido assíncrono ao servidor - usando normalmente XML como forma de representação dos dados - que trata do pedido e retorna os dados correspondentes para 0 AJAX, que por fim os mostra ao usuário (Garret 2005).

A grande diferença entre estes dois modelos, é que enquanto no modelo tradicional têm-se a necessidade de recarregar toda a página cada vez que é feito um pedido, com AJAX, uma vez que foi feito, no carregamento inicial só serão alterados os dados na área onde os mesmos serão apresentados. O fato de ser assíncrono também possibilita o processamento de outros dados enquanto se espera pela resposta do servidor.
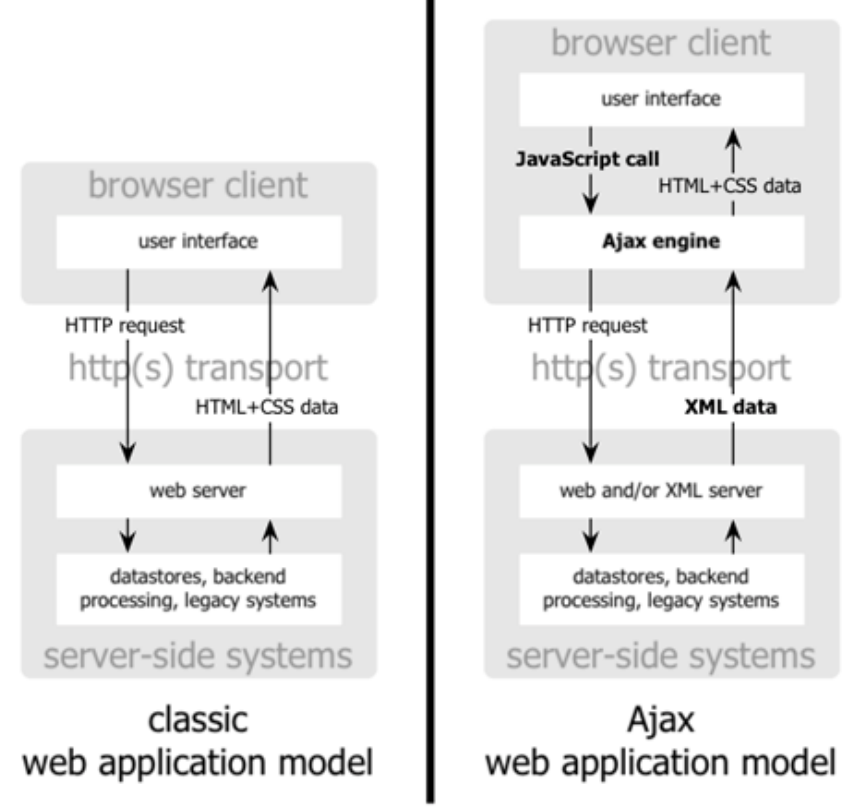

Figura 1. O modelo tradicional das aplicações Web comparado ao modelo AJAX (Garret 2005). 


\section{Proposta de Ferramenta}

Com base em informações sobre a melhor forma de desenvolvimento de aplicações que rodam na Internet e análise dos principais problemas encontrados na utilização da classe FPDF, esta seção se propõe a apresentar uma estrutura de ferramenta que possibilite ao usuário personalizar os relatórios a serem exportados em PDF através de uma interface Web.

\subsection{Projeto}

A necessidade de uma ferramenta com interface Web para a criação de relatórios no formato PDF foi o fator motivador do estudo para o desenvolvimento dessa ferramenta. Existindo então essa necessidade, o próximo passo foi fazer uma pesquisa bibliográfica para determinar quais classes ou bibliotecas em PHP que possuíam a finalidade de exportação e que conseqüentemente poderiam servir como base para essa nova ferramenta. Com base nessa pesquisa bibliográfica, a ferramenta será desenvolvida em PHP utilizando a classe FPDF e a tecnologia AJAX.

\subsection{Arquitetura}

A arquitetura implementada no modelo proposto é mostrada graficamente na figura 2. O acesso para o usuário ocorre através de requisições ao servidor Web, que interage com o a tecnologia AJAX, o qual por sua vez envia as ações ao código PHP onde se encontra a classe FPDF, que faz a consulta na base de dados e por fim monta o relatório retornando ao cliente o arquivo no formato PDF.

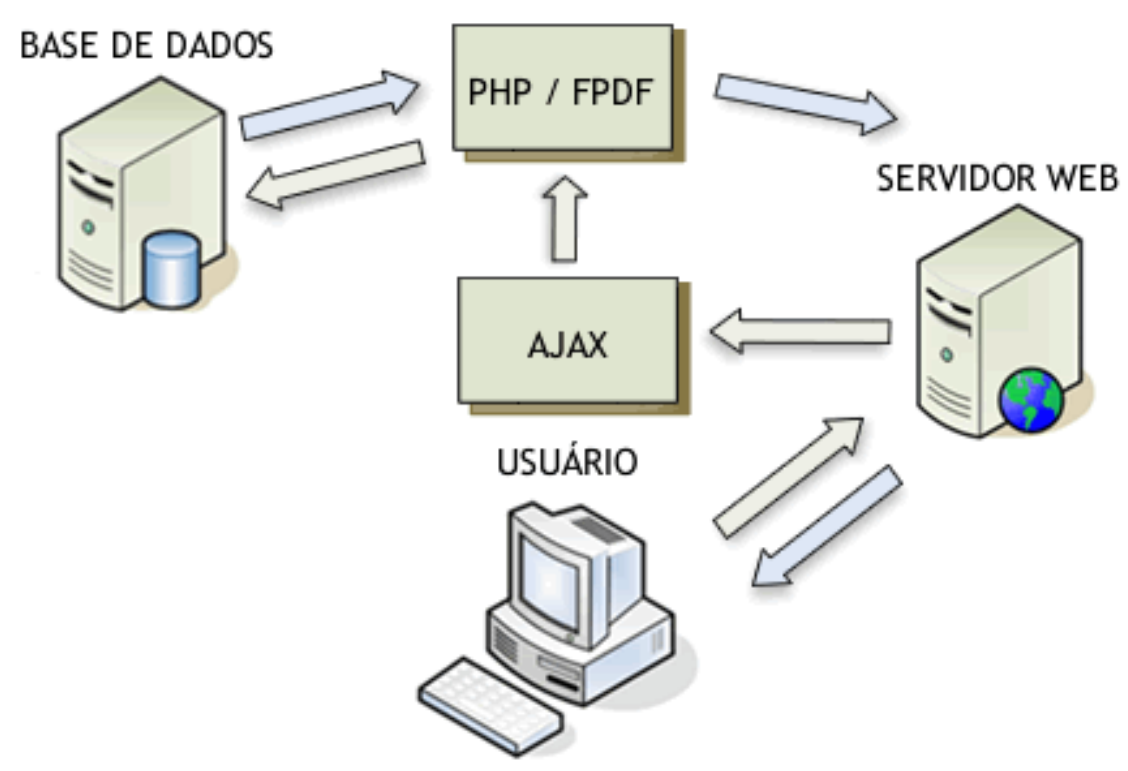

Figura 2. Arquitetura da Ferramenta.

\subsection{Interface e utilização do AJAX}

A proposta é criar uma interface intuitiva e de fácil manipulação por parte do usuário, permitindo a personalização do relatório de forma simples e ágil. Essa interface será desenvolvida utilizando XHTML e CSS, evitando assim a necessidade da instalação e 
configuração de plugins ou extensões como Flash ou Applets JAVA. Optou-se por utilizar essas linguagens, pois além de permitirem um maior nível de padronização, oferecem páginas Web mais leves e semanticamente corretas.

Outro ponto a ser destacado no que diz respeito à interface com o usuário, é a utilização da tecnologia AJAX que permite uma interação mais dinâmica entre a aplicação e o usuário. A idéia assim é proporcionar ao usuário uma navegação atrativa e rápida sem aqueles cansativos e intermináveis tempos de espera após um clique.

\subsection{Funcionamento}

Visando maior clareza e simplicidade, serão listadas as principais características do funcionamento da ferramenta:

- Possibilita ao usuário selecionar através de botões qual operação deseja executar, como por exemplo, a inclusão de um texto ou uma célula de uma tabela;

- Permite a criação personalizada de cabeçalho e rodapé do relatório;

- O usuário pode escolher o formato e a cor da fonte do texto a ser inserido no documento;

- Permite arrastar e soltar elementos de um documento;

- Ao clicar sobre um elemento permite a alteração de suas características.

\section{Considerações finais}

Este trabalho propõe o desenvolvimento de uma ferramenta gráfica para geração de relatórios no formato PDF através de uma interface Web. Esta ferramenta tem por objetivo tornar a tarefa de exportar relatórios PDF mais fácil e agradável, tanto para usuários sem conhecimentos específicos de informática, quanto para usuários com conhecimentos mais avançados.

Por apresentar uma grande facilidade de uso, a ferramenta proposta constituiu-se num ambiente com grandes perspectivas de utilização e crescimento. Dessa forma, é objetivo o desenvolvimento desta ferramenta no decorrer do trabalho de conclusão do curso de Ciência da Computação. A partir do estudo teórico realizado e descrito neste artigo, pretende-se disponibilizar uma versão beta para testes até o final do próximo ano.

\section{Referências}

ASLESON, R. e SCHUTTA, N. T. (2006) "Fundamentos do Ajax". Alta Books, Brasil.

DAUM, B. (2002) “Aprendendo XML”. Campus, Brasil.

DAVENPORT, T. H.; DICKSON, T. e MARCHAND D. A. (2000) "Dominando a Gestão da Informação”. Artmed, Brasil.

FPDF (2006) “FPDF Library”. Disponível em: http://www.fpdf.org. Acesso em: 02 de Setembro de 2006. 
GARRET, J. J. (2005) “Ajax: A New Approach to Web Applications”. Disponível em: http://www.adaptivepath.com/publications/essays/archives/000385.php. Acesso em 20 de Maio de 2006.

NEGRINO, T. e SMITH, D. (2004) “JavaScript para a World Wide Web”. Traduzido por Adriana Kramer. Campus, Brasil.

NIEDERAURER, J. (2001) “XHTML: Guia de Consulta Rápida”. Novatec, Brasil.

PDFLIB (2006) "PDFlib - A library for generating PDF on the fly”. Disponível em: http://www.pdflib.com/products/pdflib/index.html. Acesso em 02 de Setembro de 2006.

PHP (2006) “ClibPDF Functions”. Disponível em: http://www.php.net/cpdf. Acesso em: 30 de Agosto de 2006.

RAY, E. T. (2001) “Aprendendo XML”. Campus, Brasil.

R\&OS (2006) “R\&OS pdf class”. Disponível em: http://www.ros.co.nz/pdf. Acesso em: 02 de Setembro de 2006.

SAS (2006) “Consultas e Geração de Relatórios”. Disponível em: http://www.sas.com/offices/latinamerica/brazil/technologies/bi/query.html. Acesso em: 10 de Setembro de 2006.

TEAGUE, J. C. (2001) “DHTML e CSS para a World Wide Web”, Campus, Brasil. THOMSON L. e WELLING L. (2003) “PHP e MYSQL Desenvolvimento Web”. Traduzido por Edson Furmankiewicz, Campus, Brasil.

WIKIPEDIA (2006) “AJAX (Web)”. Disponível em: http://pt.wikipedia.org/wiki/AJAX_\%28Web\%29. Acesso em: 10 de Agosto de 2006.

WIKIPEDIA (2006a) “XSTL”. Disponível em: http://pt.wikipedia.org/wiki/XSLT. Acesso em 18 de Junho de 2006. 